\title{
Evidence for a role of action in colour perception
}

\author{
Aline Bompas, J Kevin O'Regan \\ Laboratoire de Psychologie Expérimentale, CNRS, Université René Descartes, 71 avenue Édouard \\ Vaillant, F92770 Boulogne-Billancourt, France; e-mail: aline.bompas@tuebingen.mpg.de, \\ oregan@ext.jussieu.fr \\ Received 29 September 2004, in revised form 11 February 2005
}

\begin{abstract}
Action is not usually considered to play a role in colour perception. However, sensorimotor theories of perception (eg O'Regan and Noë, 2001 Behavior and Brain Science 24 939-1011) suggest that, on the contrary, the transformations created by action in the sensory input are a necessary condition for all perception. In the case of colour vision, eye movements may explain how a retina with significant irregularities in resolution and cone arrangement (Roorda and Williams, 1999 Nature 397520 -522) could permit the perception of a richly coloured world (Clark and O'Regan, 2000 15th International Conference on Pattern Recognition volume 2: Pattern Recognition and Neural Networks pp 503-506; Skaff et al, 2002 16th International Conference on Pattern Recognition volume 2, pp 681-684). We provide evidence that perception of colour is modified when an artificial coupling is introduced linking eye movements and colour changes. After 40 min of wearing left-field-blue/right-field-yellow spectacles, observers' colour vision adapts so that, after removing the spectacles, white patches seem to become bluer when the eyes move rightwards and yellower when the eyes move leftwards. This induced dependence of colour perception on the direction of eye saccade is shown to be related to the amount of eye movements during exposure. This result, which cannot be explained either by retinal adaptation, or by a conditioned association between colour and side, constitutes first clear evidence for a role of eye movements in perceived colour and argues for the involvement in colour perception of neural mechanisms continuously tuned to sensorimotor contingencies.
\end{abstract}

\section{Introduction}

\subsection{The role of action in spatial properties}

The role of action in visual perception has been a subject of investigation over many years. Thus, there is no doubt that spatial properties like structure and depth are better extracted when eye and head motion is allowed (van Damme and van de Grind 1996; Dijkstra et al 1995; Rogers and Rogers 1992; Wexler et al 2001). Furthermore, for spatial properties, the link between action and perception is subject to adaptation, suggesting that vision involves a process of continuous evaluation of the laws of covariation between actions and sensory inputs. This is illustrated by studies of perceptual adaptation to optical distortions introduced by wedge prisms (Harris 1965; Held and Freedman 1963; Rock 1966; Welch 1974), in which the eye-movement-dependent shifts, and contractions and expansions of the visual field caused by the prisms are rapidly compensated.

\subsection{What is the possible role of action in colour perception?}

The role of action in the perception of non-spatial properties is less well accepted. Recent sensorimotor theories of perception claim that action should play a constitutive role (Gibson 1962; Hurley 1998; Myin 2001; O'Regan and Noë 2001) even for non-spatial properties. Colour, in particular, is often considered to be a locally defined property of the visual scene, whose determination is not expected to involve action. Colour, therefore, constitutes a particularly interesting example to test.

Motivated by different theoretical arguments, Kohler (1962) tried to induce a dependence of colour perception on eye position in the orbits, and had a single subject wear half-split coloured goggles for 60 days continuously. On removing the goggles, 
the subject showed small differences in adjusting the white of a screen, depending on whether he looked at the screen with fixed gaze oriented rightwards or leftwards. Several better-controlled studies tried to reproduce this effect (Harrington 1965; Leppmann and Wieland 1966; McCollough 1965b) - with a total of five subjects, one lasting as long as 146 days-without success and Kohler's results were attributed to nonhomogenous adaptation in peripheral retina (McCollough 1965b). Furthermore, recently, the discovery of two main streams in the visual system (Milner and Goodale 1995; Ungerleider and Mishkin 1982) - the ventral stream, devoted to object discrimination and involving form and colour; and the dorsal stream, involved in motion and space perception for action-would appear to render improbable any role of action in colour perception.

On the other hand, other arguments suggest a necessary involvement of action in colour. Colour perception in humans is classically defined in terms of relative excitation of three classes of cones, the $\mathrm{L} / \mathrm{M} / \mathrm{S}$ ratio. However, most often, this ratio cannot be defined: in a normal retina, large patches lack at least one class of cones (Roorda and Williams 1999), without this giving rise to perceptual nonhomogeneity in everyday life. An explanation of this paradox might lie in eye movements. Eye movements allow the same surface patch to be exposed to many areas in the retina and it may therefore be necessary for the $\mathrm{L} / \mathrm{M} / \mathrm{S}$ ratio to be well defined. Eye movements could also help to improve colour judgments by exploiting retinal nonhomogeneity due to macular pigment and by allowing differently illuminated areas of a coloured surface to be explored (Clark and O'Regan 2000; Skaff et al 2002). These last two facts have been proposed by Broackes (1992) as reasons why dichromats, whose retina possesses only two classes of cones, perform better than they should according to classical models of colour perception (Jameson and Hurvich 1978).

These arguments are consistent with an alternative definition: colour could be specified by the transformations in cone excitation created by eye movements or, more precisely, by a learnt coupling between sensation and action. A first step towards finding experimental support for this idea would be to show that the modification of this coupling results in a modification of colour sensation. This is the subject of the present study.

\subsection{Relation to previous experiments}

We based ourselves on Kohler's adaptation paradigm: exposure to left-field-blue/ right-field-yellow spectacles (figure 1), preceded and followed by a test stage without them. However, our approach was different from Kohler's or McCollough's. Theirs and other previous experiments reported in the literature were performed to investigate the possibility of 'conditioning' colour sensation by gaze direction. In this 'situational conditioning', eye position was considered as proprioceptive information that could be used as a cue for the perceptual system to decide when it had to compensate for yellow or blue. Our approach is fundamentally different and led us to adopt a different methodology. First, we wanted to show that vision is based on continuous analysis of sensorimotor regularities. This led us to study short-term mechanisms, both for adaptation and measures. Second, our concern was not to look for possible interactions or conditioning between vision and static proprioceptive information, but instead to consider vision as resulting from the activity of a dynamic perceptual system which includes ongoing muscle commands. Our experiments were motivated by the hypothesis that the perceptual learning we induced is a manifestation of a sensorimotor coupling at work in the normal vision process. Our conception therefore predicted that perceptual learning occurs only through eye movement and led us to focus on eye movements instead of eye position in the orbit. Similarly, instead of measuring the 
aftereffect by a static method, as has been done so far, we measured the perceptual changes that occurred across the saccade in a comparison task.

To avoid the criticism of Kohler's work, several precautions had to be taken. During exposure, eye movements ensured equal adaptation of the central retina to blue and yellow. However, as noted by McCollough (1965b) in a criticism of Kohler, this was not true of the peripheral retina. This nonhomogeneity of adaptation could have created contrast effects between central and peripheral retina during the test phase that Kohler's procedure failed to prevent. First, exposure was made with goggles that limited the visual field and the frame was kept for test stages. This created a bias, since, depending on the direction of gaze, different regions of the retina were masked and therefore unused during white adjusting. This is why we preferred to use spectacles and perform the test stages without the spectacle frame. Moreover, Kohler's test stages used a whole white screen, therefore stimulating the totality of the non-masked retina. We used, instead, for the test phase small patches on a black screen, involving only the central retina, supposed to be homogeneously adapted.

\section{General methods}

\subsection{Display}

The stimuli were generated on a PC with Matlab with the psychophysics toolbox extension (Brainard 1997; Pelli 1997). They were coded with 24-bit precision and presented on a CRT display (NVidia Vanta, $80 \mathrm{~Hz}$ refresh). The subject's head was fixed in a chin-rest. The viewing distance was $35 \mathrm{~cm}$. Luminance and chromaticity coordinates were measured with a Minolta CS-100 photometer with close-up lens no 110. The experiments were conducted in a lighted room and all the stimuli were presented on a black background $\left(L=0.03 \mathrm{~cd} \mathrm{~m}^{-2}\right)$.

\subsection{Materials}

The spectacles were designed so that, when fixating a point at a distance of $40 \mathrm{~cm}$, the visual hemifield situated on the left of this point was tinted in yellow, while the right visual hemifield was tinted in blue (as can be seen in figure 1, this bipartition of the visual field is not achieved by simply splitting each lens into two equal surfaces). The coloured glasses were specially made and their properties were quantified by measuring the chromaticity coordinates of a white patch seen through the blue and the yellow glasses (figure 2). The distance $\mathrm{Y}_{\mathrm{g}}-\mathrm{B}_{\mathrm{g}}$ was the colour difference between a white patch $(x=0.264, y=0.300)$ seen through the yellow glass $\left(\mathrm{Y}_{\mathrm{g}}: x=0.419\right.$, $y=0.524)$ and seen through the blue glass $\left(\mathrm{B}_{\mathrm{g}}: x=0.189, y=0.225\right)$, relative to the chromaticity coordinates in the CIE 1931 system. When wearing the spectacles and executing an eye saccade from left to right (respectively from right to left), the subject experienced a mean colour change of $Y_{g}-B_{g}$ (or $B_{g}-Y_{g}$ ). Therefore, the amount of adaptation was expressed as a percentage of $\mathrm{Y}_{\mathrm{g}}-\mathrm{B}_{\mathrm{g}}$.

\subsection{Task and procedure for test stages}

The test stage described here remained identical in all experiments, with the exception of experiment 5. To measure the effect of adaptation, the direction of eye saccade and colour changes were manipulated. Each test trial involved successive presentation of two patches, either first on the left and then on the right side of the screen (LR), or in the right-to-left order (RL). The colour set involved five unsaturated colours around neutral grey, chosen between $\mathrm{Y}_{\mathrm{g}}$ and $\mathrm{B}_{\mathrm{g}}$. $\mathrm{G}$ was the grey whose luminance was half-way between $\mathrm{Y}_{\mathrm{g}}$ and $\mathrm{B}_{\mathrm{g}}$. B1 and $\mathrm{B} 2$ were two different blues at $5 \%$ and $10 \%$ of $\mathrm{G}-\mathrm{B}_{\mathrm{g}}$. Y1 and $\mathrm{Y} 2$ were two different yellows at $5 \%$ and $10 \%$ of $\mathrm{G}-\mathrm{Y}_{\mathrm{g}}$. The five colour changes involved in the test stages were $\mathrm{B} 2-\mathrm{Y} 2, \mathrm{~B} 1-\mathrm{Y} 1, \mathrm{G}-\mathrm{G}, \mathrm{Y} 1-\mathrm{B} 1$, and $\mathrm{Y} 2-\mathrm{B} 2$, corresponding respectively to $-10 \%,-5 \%, 0 \%,+5 \%$, and $+10 \%$ of the colour change $\mathrm{Y}_{\mathrm{g}}-\mathrm{B}_{\mathrm{g}}$. Patches were $8.4 \mathrm{deg}$ wide, centred at $6.4 \mathrm{deg}$ eccentricity (on the left or on 


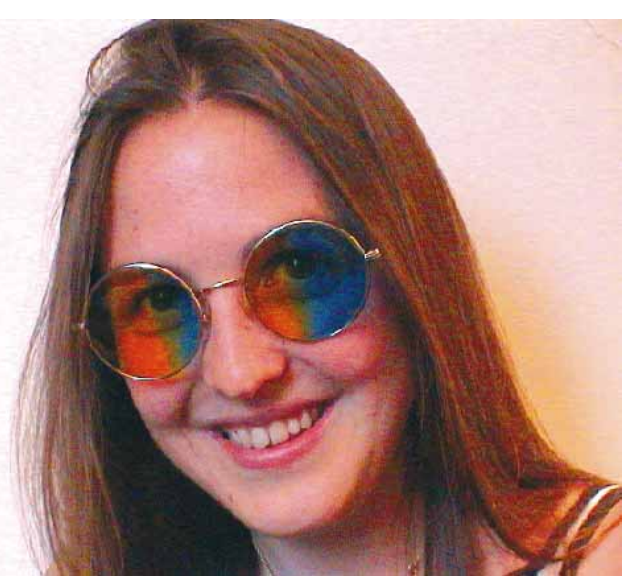

Figure 1. Subject wearing half-split coloured spectacles.

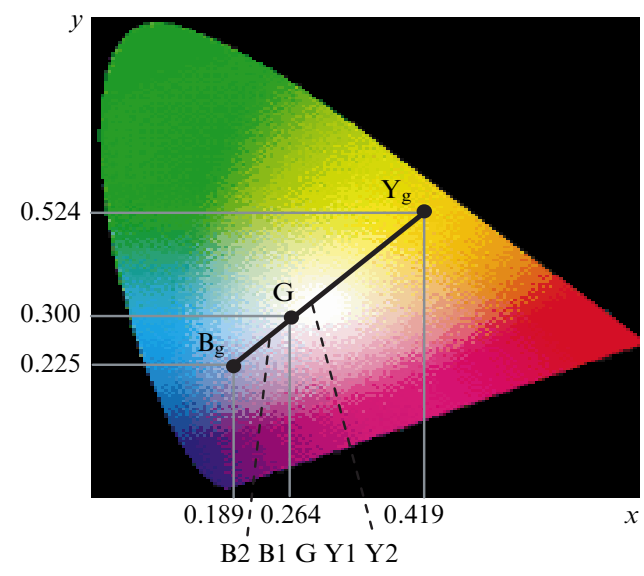

Figure 2. Chromaticity coordinates of glasses and colour set in the test stage.
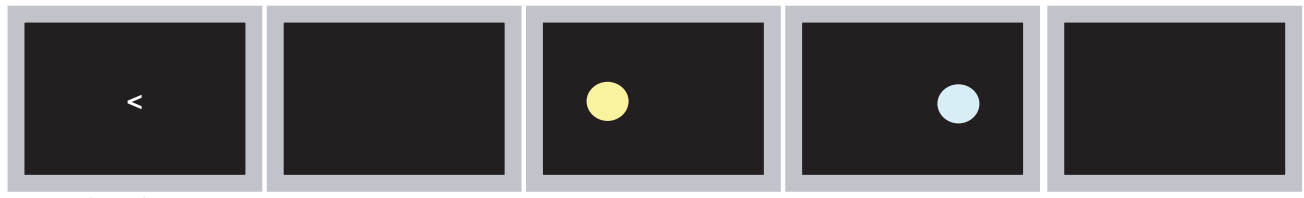

LR, Y2-B2
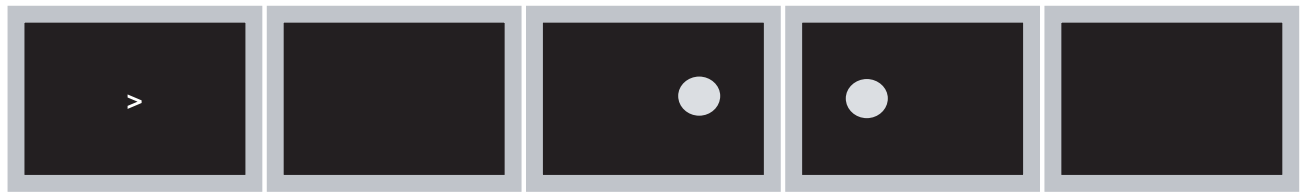

RL, GG

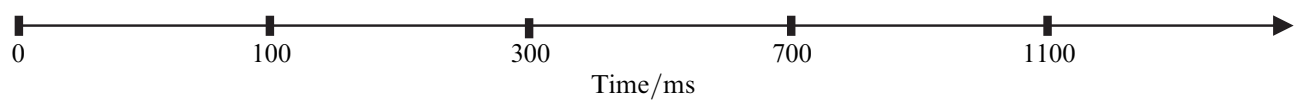

Figure 3. The time sequence in the dynamic test stage. Top: left-right trial with the larger colour change from yellow to blue (Y2-B2), corresponding to $+10 \%$ of $\mathrm{Y}_{\mathrm{g}}-\mathrm{B}_{\mathrm{g}}$. Bottom: right - left trial without colour change (the two patches are grey: $\mathrm{G}-\mathrm{G}$ ), corresponding to $0 \%$ of $\mathrm{Y}_{\mathrm{g}}-\mathrm{B}_{\mathrm{g}}$.

the right) and were presented for $400 \mathrm{~ms}$. The time sequence of the test trials is illustrated in figure 3: an arrow indicated the side of the first patch, whose appearance was immediately followed by the second patch. The subjects' task was to determine if the second patch was more blue or more yellow than the first patch. The subjects responded by pressing the upper and lower keys. Each colour change was presented 18 times in each of the left-right (LR) and the right-left (RL) orders. All the trials were presented randomly except for the first trial (which was always $L R, G-G$ ) and the second one $(\mathrm{RL}, \mathrm{G}-\mathrm{G})$. The subjects were asked to pay special attention to the first two trials, which could be repeated at will, by pressing the left or right key, until they felt able to answer with certainty. This allowed us to obtain a crude indication of the existence of an aftereffect just after removal of the spectacles, in case no measurable effect could be obtained through the whole test stage.

\subsection{Predictions}

During exposure with the spectacles, left-right (respectively right-left) eye saccades were correlated with a colour change from yellow to blue (respectively from blue to yellow). 
If the visual system adapts-even partially - to this correlation, after the subject has removed the spectacles, the effects of an LR and RL eye movement were expected to be opposite. If adaptation occurs between the two test stages, the proportion of "more yellow" responses would be expected to increase in LR trials and to decrease in RL trials. Adaptation was established when (i) differences between test stages before and after adaptation were obtained and (ii) these differences were opposite for LR and $\mathrm{RL}$ trials, pointing in the same direction as our predictions.

\section{Experiment 1: $4 \mathrm{~h}$ free exposure}

\subsection{Observers}

Three non-naive subjects, including one of the authors and two students, participated in the first experiment. During free exposure with the spectacles, they had no particular task and moved their eyes normally while executing everyday activities - such as reading, walking, and talking $-4 \mathrm{~h}$ a day (F4h). The experiment was conducted for 4 days for each subject, with 2 days in a row, 1 day stop and 2 days in a row again, to investigate possible cumulative effects.

\subsection{Results}

From subjects' responses to the first two trials, just after they had removed the spectacles at the end of exposure, two identical grey patches were perceived as different, in a way that depended on the direction of eye movement and compensated for the effect of the spectacles. Thus, just after the exposure stage, when shown two identical grey patches in the left-right order (LR), all the subjects perceived the second patch as more blue than the first one. For right-left order (RL), all the subjects reported the second one as being more yellow.

Differences in the proportion of "more yellow" responses between pre-test $\left(t_{0}\right)$ and post-test $\left(t_{1}\right)$ were calculated for each subject, day, colour change, and direction. For each subject, the mean differences on the 4 days introduced by exposure are presented as a function of the colour change in figure 4 . All the colour changes were not affected equally by exposure: in particular, large colour changes $\left(-10 \%\right.$ and $\left.+10 \% \mathrm{Y}_{\mathrm{g}}-\mathrm{B}_{\mathrm{g}}\right)$ were less affected, indicating that they remained correctly perceived even after exposure. However, the global effect of exposure was clear: positive differences in the percentage of "more yellow" responses were observed for left - right eye movements, whereas these differences were negative for right-left trials. Colour judgments were modified by exposure in accordance with our predictions and opposite effects were observed for LR and RL directions.

To quantify the effect and calculate its global significance, we fitted the percentage of "more yellow" responses as a function of the colour change with a sigmoid and obtained the point of subjective equality (PSE), as illustrated on a single subject in figure 5. The PSE is interpreted as the physical colour change that would be perceived as nonchanging by the subject. The PSE shift is the difference between pre-test and post-test and quantifies the effect of the exposure stage. For each subject, the mean PSE shift on the four measures is compared for LR and RL (figure 6). All subjects showed significant differences between LR and RL PSE shifts (CC: $F_{1,3}=19.93$, $p=0.021$; AB: $F_{1,3}=18.07, p=0.024$; SG: $\left.F_{1,3}=170, p=0.001\right)$. For LR eye movements, the mean PSE shift for the three subjects is positive $\left(F_{1,11}=10.51, p=0.008\right)$, indicating that subjective equality is obtained for yellow to blue changes (positive percentage of $Y_{g}-B_{g}$ ), ie for the type of changes imposed by the spectacles when moving the eyes from left to right. Conversely, the mean PSE-shift for RL is negative $\left(F_{1,11}=55, p<0.0001\right)$. This confirms that perceptual learning has occurred, the difference between blue and yellow glasses having been partly compensated. 

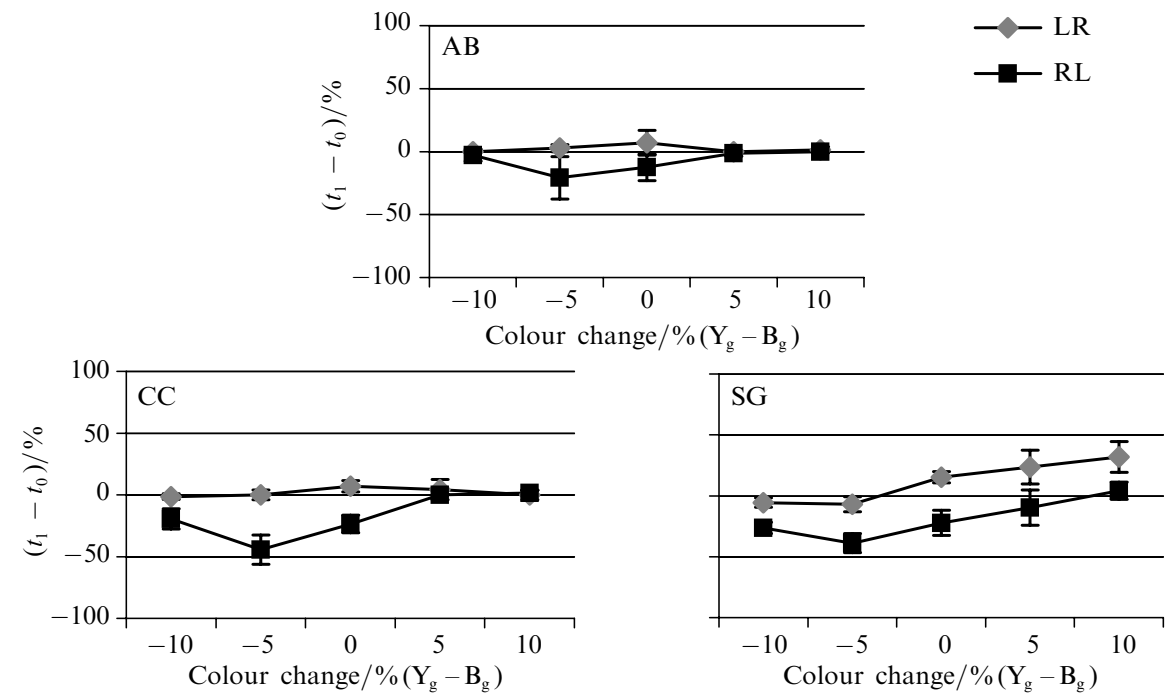

Figure 4. Experiment 1. Effect of exposure is measured by the difference in the percentage of "more yellow" pre-test responses $\left(t_{0}\right)$ and post-test responses $\left(t_{1}\right)$. The effects of a $4 \mathrm{~h}$ free exposure are presented for each of the three subjects (AB, CC, and SG) as a function of colour change for left-right (LR) and right-left (RL) eye movements. Error bars indicate one standard deviation on either side of the mean calculated across the 4 days.

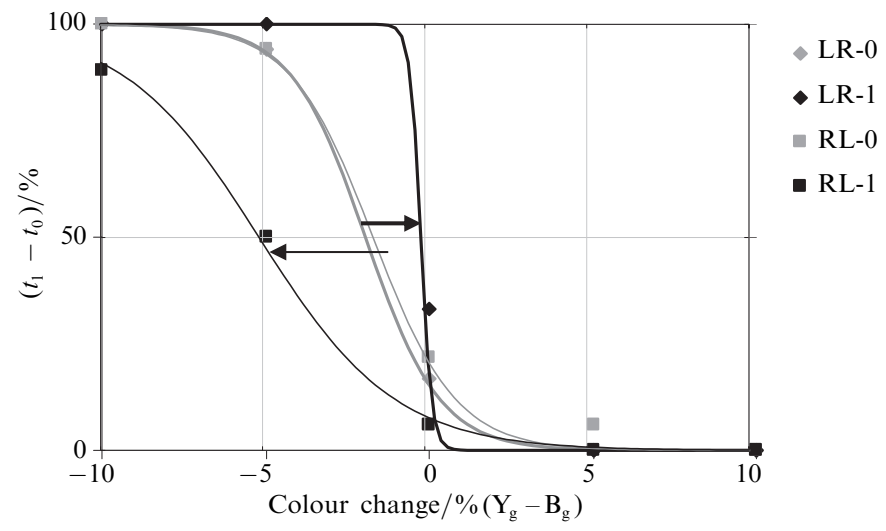

Figure 5. Illustration of the PSE-shift determination on subject $A B$ on the first day. Data points represent the percentage of "more yellow" responses $\left(t_{1}-t_{0}\right)$ for the five colour changes used corresponding to $-10 \%,-5 \%, 0 \%,+5 \%$, and $+10 \%$ of $\mathrm{Y}_{\mathrm{g}}-\mathrm{B}_{\mathrm{g}}$. Data (points) are fitted with sigmoid (lines) for left-right (LR, thick) and right-left (RL, thin), before (0, grey) and after (1, black) exposure. The PSE is the colour change corresponding to $50 \%$ "more yellow" responses. The PSE shift is the difference in PSE between $t_{0}$ and $t_{1}$, represented by the arrows.

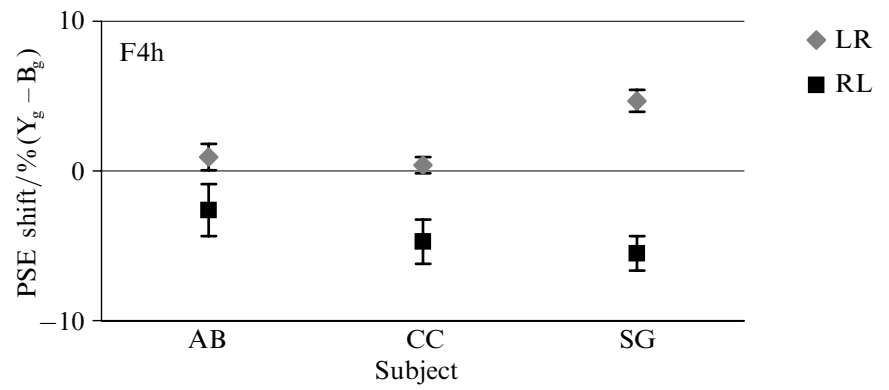

Figure 6. Experiment 1. Mean PSE shifts for left-right (LR) and right-left (RL) eye movements induced by $4 \mathrm{~h}$ of free exposure, for each of the three subjects. 


\subsection{Discussion}

Measurable adaptation was obtained after only $4 \mathrm{~h}$. It is of some interest to compare it with the several months thought to be necessary by previous investigators. However, adaptation is consistent but rather weak, since it is never larger than $5 \%$ of $Y_{g}-B_{g}$, the mean effect being 3.15\%. Subjective impressions during wearing of the spectacles were not systematically recorded. However, even if subjects spontaneously reported being less disturbed by the spectacles, the colour difference remained clearly visible during the whole exposure. In addition, no cumulative effect was measurable from one day to the next, giving little hope of obtaining a larger effect with longer exposure duration. Moreover, differences between individuals led us to perform an even shorter experiment, so that the effect could be tested on more subjects-rather than trying to obtain a larger effect on a very small number of subjects-and to look for the conditions that permitted to maximise adaptation in $40 \mathrm{~min}$.

\section{Experiment 2: $40 \mathrm{~min}$ free exposure}

\subsection{Observers}

Eight observers, including seven naive subjects, participated in the second experiment, in the same conditions as in the first experiment but with the exposure limited to $40 \mathrm{~min}$ and occurring only once per subject (F40m).

\subsection{Results}

As can be seen in figure 7, no clear differences between $t_{0}$ and $t_{1}$ were observed, as confirmed by the calculated PSE shifts (figure 8). In LR condition, the mean PSE shift is approximately 0 , showing that the exposure stage had no effect $\left(F_{1,7}=0.11\right.$, $p=0.745)$. In RL condition, the negativity of the mean PSE shift is only just significant $\left(F_{1,7}=5.63, p=0.049\right)$ and is related to a very small effect $\left(1.36 \%\right.$ of $\left.\mathrm{Y}_{\mathrm{g}}-\mathrm{B}_{\mathrm{g}}\right)$. This absence of adaptation is confirmed by the non-significance of the mean difference in PSE shifts between LR and RL directions $\left(F_{1,7}=1.65, p=0.240\right)$.

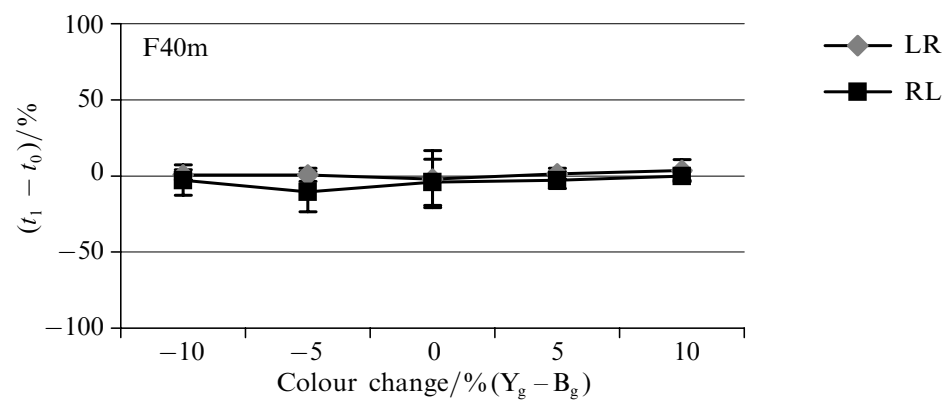

Figure 7. Experiment 2. Mean effect of a $40 \mathrm{~min}$ free exposure as a function of colour change for left - right (LR) and right - left (RL) eye movements. Error bars indicate one standard deviation on either side of the mean calculated across the eight subjects.

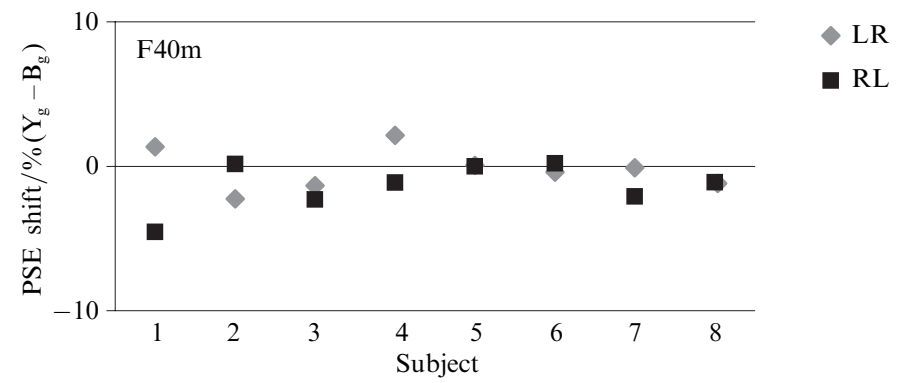

Figure 8. Experiment 2. PSE shifts for left-right (LR) and right-left (RL) eye movements induced by $40 \mathrm{~min}$ of free exposure, for each of the eight subjects. 


\subsection{Discussion}

The most obvious explanation for the differences between the first two experiments is the amount of time given to the visual system to learn the new contingency. However, to affirm this point, we must exclude at least two alternative interpretations. The first one would attribute the success of the first experiment to purely retinal adaptation. Despite all the precautions we took to limit the bias (see section 1.3), we cannot totally exclude this interpretation. Another explanation for the difference between the first two experiments could be the level of motivation or attention paid to colour by the first three, non-naive, subjects in experiment 1.

The only way to get definitively rid of the 'purely retinal' interpretation was to design an exposure stage that allowed an effect to be obtained without increasing the nonhomogeneity in retinal adaptation. According to us, adaptation occurs through eye movements and the more the visual system has the opportunity to produce appropriate sensorimotor transformations, the more it learns it. We therefore designed a 'constrained' exposure stage (C), of the same duration as in experiment 2, where the subjects, while wearing the spectacles, were given a task that needed repeated eye movements from left to right, namely comparison between two patches appearing on the left and right sides of a computer screen. To investigate whether attention to colour during adaptation plays a role, the patches could change both in colour and in size: half of the subjects had to compare colour (Cc) while the others focused on size (Cs).

\section{Experiment 3: Short-lasting constrained exposure}

\subsection{Observers}

Twenty-three subjects participated in experiment 3 , fourteen in condition $\mathrm{Cc}$ and nine in condition Cs. Three subjects in condition $\mathrm{Cc}$ and one subject in condition Cs were excluded because their psychometric function slopes were below 0.55 , corresponding to $75 \%$ correct for the larger changes (Y2-B2 and $\mathrm{B} 2-\mathrm{Y} 2$ ) for a non-biased curve, indicating they had difficulties in doing the colour discriminations necessary to determine the PSE.

\subsection{Task and procedures in constrained exposure stages}

The time sequence of the task given to the subjects while wearing the spectacles was similar to the time sequence of the test stages (figure 9): a central arrow indicated the side of the first patch, whose appearance was immediately followed by the second patch. The two patches could differ both in colour and in size. Subjects decided whether the two patches were identical or different-with respect to colour in condition $\mathrm{Cc}$ and to size in condition $\mathrm{Cs}$ - and received feedback. Colour pairs were randomly chosen from a colour set containing two saturated blues, two saturated yellows, and a neutral grey. The colour set was chosen along the blue-yellow axis, supposed to be the most strongly affected by the colour transformation induced by the yellow and blue filters. The size of the patches could be either 8.4 or $9 \mathrm{deg}$, both presented at $6.4 \mathrm{deg}$ eccentricity. The exposure lasted for $40 \mathrm{~min}$, preceded and followed by the same test stages as in the previous experiments.

\subsection{Results}

Clear differences between $t_{0}$ and $t_{1}$ can be observed in both condition $\mathrm{Cc}$ and $\mathrm{Cs}$ (figure 10). These differences are positive for the LR condition, which is confirmed by the significantly positive PSE shifts (Cc: $F_{1,10}=9.97, p=0.010$; Cs: $F_{1,7}=30.19$, $p=0.0009$ ), as can be seen in figure 11. Observed differences are negative for RL (figure 10), as confirmed by significantly negative PSE shifts $\left(\mathrm{Cc}: F_{1,10}=8.17, p=0.017\right.$; Cs: $\left.F_{1,7}=43.93, p=0.0006\right)$ in figure 11. In both conditions $\mathrm{Cc}$ and $\mathrm{Cs}$, significant opposite PSE shifts are obtained for LR and RL eye movements (Cc: $F_{1,10}=10.32$, $p=0.009$; Cs: $\left.F_{1,7}=59, p=0.0001\right)$. No significant difference is found between PSE 

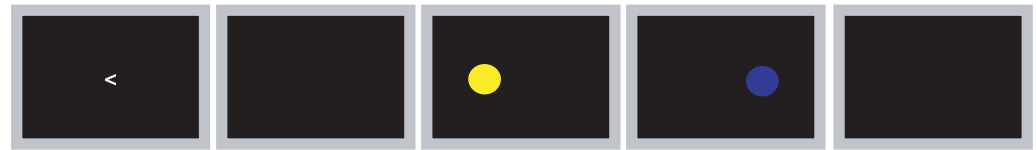

LR, colour change, no size change
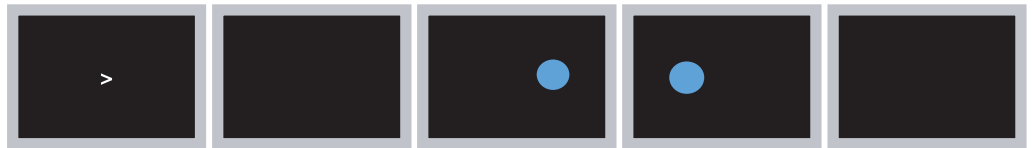

RL, no colour change, size change

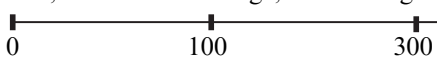

300 700

Time $/ \mathrm{ms}$

Figure 9. Time sequence in constrained exposure.
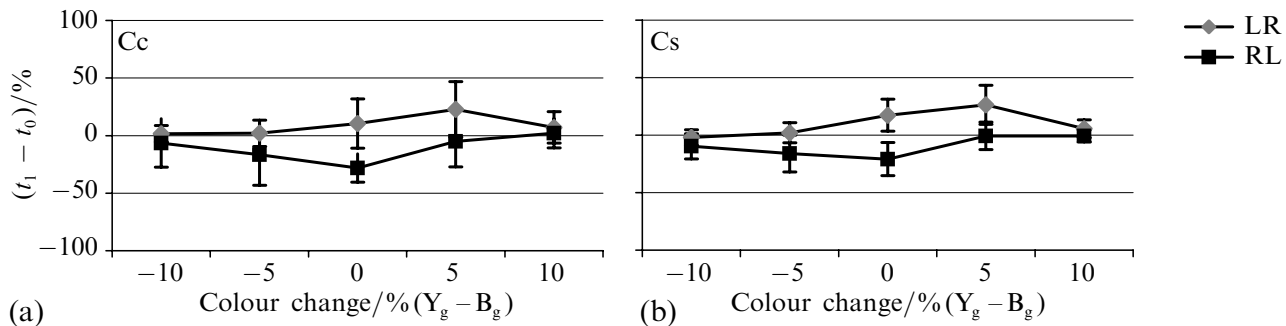

Figure 10. Experiment 3. Mean effect of a 40 min constrained exposure with repeated eye movements as a function of colour change for left-right (LR) and right-left (RL) eye movements. In condition Cc, eleven subjects were involved in a task on colour during exposure (a). In condition Cs, eight subjects were involved in a task on size during exposure. Error bars indicate one standard deviation on either side of the mean calculated across the subjects (b).

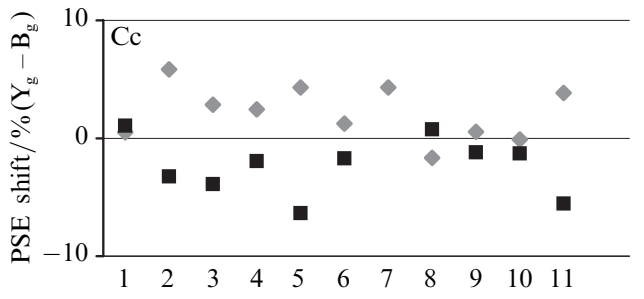

(a) (b)

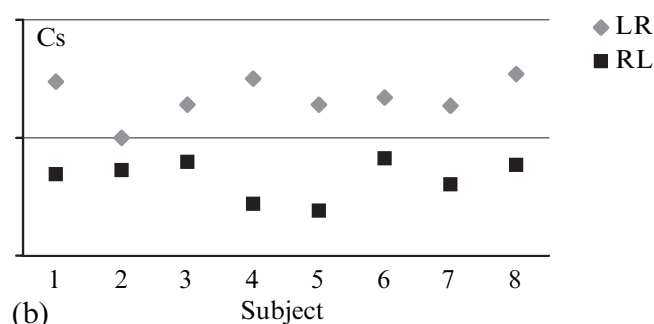

Subject

Figure 11. Experiment 3. PSE shifts for left - right (LR) and right - left (RL) eye movements induced by $40 \mathrm{~min}$ of constrained exposure. Results for eleven subjects are presented in condition Cc (a) and eight in condition Cs (b).
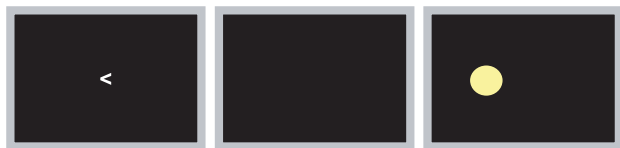

$\mathrm{L}, \mathrm{Y} 2$
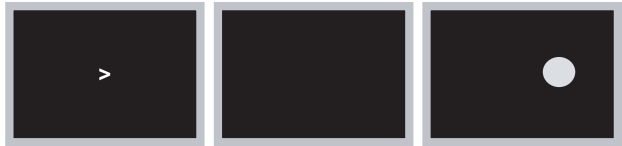

$\mathrm{R}, \mathrm{G}$

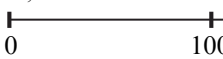

100
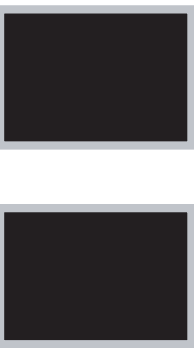

Time $/ \mathrm{ms}$

Figure 12. Time sequence of static test stages involved in experiment 4 . 
shifts in conditions $\mathrm{Cc}$ and $\mathrm{Cs}$, either for $\operatorname{LR}\left(F_{1,17}=1.45, p=0.25\right)$ or for $\mathrm{RL}$ $\left(F_{1,17}=0.01, p=0.90\right)$.

\subsection{Discussion}

It seems that, thanks to repeated eye movements during exposure, significant adaptation to the spectacles was obtained in $40 \mathrm{~min}$, while no such effect was obtained with free exposure of the same duration in experiment 2. This success excludes the 'purely sensory' interpretation based on nonhomogeneity of adaptation in the peripheral retina. This is even more obvious when one considers that this nonhomogeneity was probably larger in the free exposure than during the constrained exposure. The surface of nonhomogeneously adapted retina depends on the eccentricities reached by the eye and the lateral amplitude of eye saccades. Since eye eccentricities reached in the unconstrained adaptation phase were very likely smaller than in the constrained adaptation phase (Rayner 1984), such a retinal interpretation would have predicted a stronger effect in the free condition. Moreover, since no difference was observed between conditions $\mathrm{Cc}$ and $\mathrm{Cs}$, the amount of attention paid to colour appears not to be relevant, at least for such a short exposure. Consequently, we conclude that a true sensorimotor adaptation for colour perception occurred, showing that the visual system is able to rapidly learn new sensorimotor contingencies between eye movements and their sensory consequences.

To understand why our attempt succeeded in $4 \mathrm{~h}$ where previous attempts failed with adaptation periods of several weeks, a single explanation remains: the test stage we used was more appropriate than the one used in previous studies. Our test stage differed from the previous one in at least three aspects. First, instead of white adjusting, we used a two-alternative forced-choice with constant-stimuli procedure. It could be that this method was more precise and revealed a weaker effect. Second, experiments were performed on several subjects, and/or with several measures per subject, in a way that permitted statistical analysis, which allowed us to detect the presence of a significant adaptation, even for very small effects. Finally, our test involved a colour comparison across eye movement, instead of an absolute colour judgment with gaze fixed rightwards or leftwards.

This last point is, in our view, the most interesting one since it illustrates the dichotomy between the 'situational conditioning' conception of previous studies and the sensorimotor approach. According to the conditioning approach, our results could be understood as the induction of a colour bias as a function of gaze direction: the visual system would add blue when gaze is directed leftwards and yellow when gaze is directed rightwards, with these effects adding together when an eye movement is performed. This would mean that the effect is a purely sensory phenomenon correlating retinal and proprioceptive inputs.

To show that action during test phase was the key factor that permitted us to reveal the sensorimotor adaptation, we designed a control experiment where the exposure stage was identical to condition Cs and the test stage used the same colour set as before, but involved absolute judgment of colour on the left and right sides.

\section{Experiment 4: Short-lasting constrained exposure with static test}

\subsection{Observers}

Fourteen subjects participated in this experiment. Five subjects were excluded on the basis of the same criterion as in experiment 3 with a constrained exposure of $40 \mathrm{~min}$, preceded and followed by static test stages $\left(\mathrm{Cs}^{\prime}\right)$.

\subsection{Task and procedure in static test}

The test stage involved the same set of five unsaturated colours: B2, B1, G, Y1, Y2. The time sequence, illustrated in figure 12 , was as close as possible to the previous one, 
with the difference that a single patch appeared on the left $(\mathrm{L})$ or on the right $(\mathrm{R})$. The subject's task was to classify the colour of the patch as yellow or blue. This static test stage was performed before and after constrained exposure with size comparison. It must be noted that the static test does not involve a comparison task and therefore does not permit to define a PSE. Instead, what we obtain is the point of subjective grey (PSG) along the axis defined by $\mathrm{B} 2-\mathrm{G}-\mathrm{Y} 2$.

\subsection{Results}

As can be seen in figure 13, the differences in colour judgment between $t_{0}$ and $t_{1}$ were absent for the five colours presented, as confirmed in figure 14 by the absence of PSG shifts for $\mathrm{L}\left(F_{1,8}=0.78, p=0.79\right)$ and $\mathrm{R}\left(F_{1,8}=3.69, p=0.091\right)$ patches, and also by the non-significance of the difference between $\mathrm{L}$ and $\mathrm{R}\left(F_{1,8}=2.61, p=0.145\right)$.

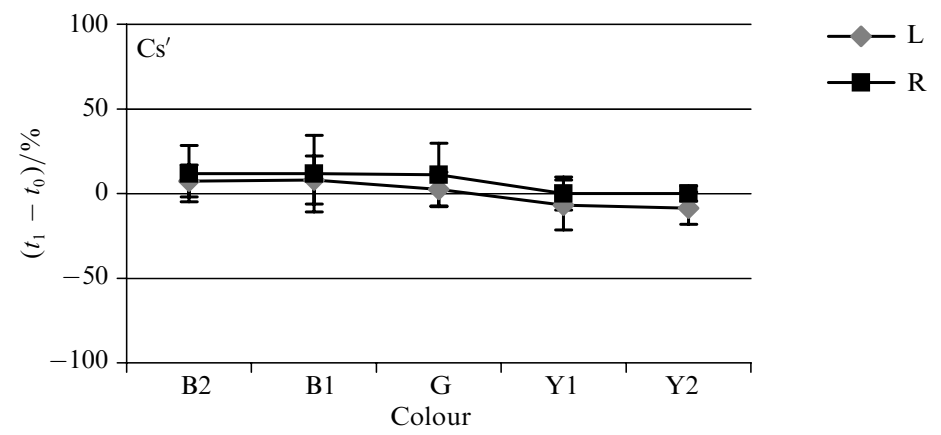

Figure 13. Experiment 4. Mean effect of a $40 \mathrm{~min}$ constrained exposure is measured by the difference in the percentage of "yellow" pre-test responses $\left(t_{0}\right)$ and post-test responses $\left(t_{1}\right)$ as a function of absolute colour for left (L) and right (R) sides. Error bars indicate one standard deviation on either side of the mean calculated across the subjects.

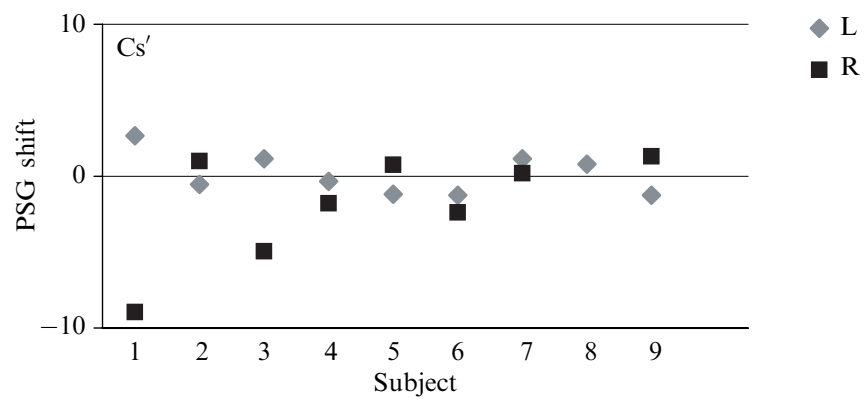

Figure 14. Experiment 4. PSG shifts for left (L) and right (R) sides induced by 40 min of constrained exposure, for each of the trial subjects.

\subsection{Discussion}

The failure to measure any effect with a static test confirms the necessity of eye movements during the test stage. Accordingly, the effect cannot be understood as the cumulative effect of a conditioned colour compensation contingent on eye position. We can therefore exclude the interpretation that the adaptation we induced was based on the interactions of different sensory mechanisms, as is the case for many effects, the most famous being McCollough's (1965a), inducing colour aftereffect contingent on orientation. Other similar studies have addressed the induction of a colour aftereffect on the direction of movement (Hepler 1968) or the induction of a movement aftereffect on the gaze direction (Mayhew 1973). Though it could be conceived of as an instance of a contingent aftereffect, the present phenomenon differs from most of those previously found-excluding perceptual adaptation to prisms or inverting mirrors- 
by the fact that it involves sensorimotor mechanisms, coupling actions, and sensory consequences of action.

It could be argued that our effect could nevertheless be described as a conditioned colour compensation, but contingent on the direction of eye movement. However, this model is unable to propose an interpretation for the fact that colour perception can be made dependent on the direction of eye movements but not on eye positions. According to this conditioning model, eye position and direction of eye movement are certainly two forms of 'information' that should be a priori equally able to serve as a signal for conditioned compensation. Consequently, the conditioning model would appear to be inadequate. On the contrary, the sensorimotor approach proposes that perception is based on the analysis of the interaction between sensors and the environment thanks to active exploration. Therefore, in this approach eye position and direction of eye movement have a different status and only the latter allows the nonhomogeneities in both retina and lighting conditions to be made use of.

It could be argued that, even in the test stage used in experiment 4, eye movements were also involved since, in each trial, the subjects moved the eyes from the central arrow to the single colour patch in periphery to make the colour judgments. Two compatible explanations can be proposed for the failure to obtain colour shift in these conditions. First, the effect could involve only mechanisms sensitive to colour temporal contrasts, having no effect on absolute judgments concerning single colours. Second, the size of the saccades in the test stage is half that in the exposure stage. Future work should deal with the dependence of the effect on these types of saccadic properties.

\section{General discussion}

Our methods produced a highly reproducible perceptual change that cannot be interpreted as a purely physiological adaptation of cone sensitivity. These results argue for the existence of mechanisms continually updated in response to current visuo-motor dependences. ${ }^{(1)}$ Since the existence of sensorimotor adaptation for colour was proposed as a necessary condition for our approach, the present results, which were predicted from this approach, can be considered a first step that makes sensorimotor approach to colour perception plausible.

Candidates for the neural implementation of such mechanisms are numerous. More and more studies have been devoted recently to the influence of eye movements on the activity of visual neurons. In particular, in the lateral geniculate nucleus (LGN), the earliest stage of visual processing after the retina, saccadic eye movements have been shown to modulate the strength and temporal properties of the visual response of many neurons (Reppas et al 2002). It is of interest that it is now established that, contrary to what had been believed so far, this modulation concerns not only the magnocellular pathway, sensitive to luminance contrasts, but also the parvocellular neurons, sensitive to colours. In many higher visual areas the activity of neurons is enhanced when stimuli presented in their classical receptive fields are targets for eye movements (Fischer and Boch 1981). Specifically, in V4, before the initiation of a saccadic eye movement, receptive fields shrink and shift towards the saccade target (Tolias et al 2001). This dependence of the activity of visual neurons on eye movements could be a plausible basis for a mechanism linking action and sensory consequences of action in an ongoing fashion.

The spectacles created a consistent bias between the observer's perceptual system and the real world. It might have been thought that pressure to adapt would have

(1) An alternative hypothesis could be that, both saccades and colour representation being predominantly organised contralaterally, learning effects could coexist simultaneously in the two cerebral hemispheres. This hypothesis would be confirmed by a failure to adapt to up/down instead of left/ right contingency. 
been stronger in the free situation where the subject was confronted with the real world and where there was knowledge about the world and about the correspondence between colours seen on the right or left. Since no effect was found in the free condition compared with the constrained conditions of the same duration, we must conclude that, at least for short exposure stages, the use of natural viewing conditions is not of paramount importance. If this is true, we predict that the same adaptation might be produced with a computer-controlled experiment, introducing a link between certain eye movements and certain colour changes, without the use of the spectacles. Such experiments will be the object of future work and may allow us to investigate more freely the parameters of this phenomenon.

Acknowledgments. We thank Cyril Charron, Andréi Goréa, James Joseph Clark, Françoise Viénot, Erik Myin, Feisal Benhassel, and Justin Broackes for comments and suggestions.

\section{References}

Brainard D H, 1997 "The psychophysics toolbox" Spatial Vision 10433 - 436

Broackes J, 1992 "The autonomy of color", in Reduction, Explanation and Realism Eds K Lennon, D Charles (Oxford: Oxford University Press) pp $421-465$

Clark J J, O'Regan J K, 2000 "A temporal-difference model of perceptual stability in color vision", 15th International Conference on Pattern Recognition volume 2: Pattern Recognition and Neural Networks pp 503-506

Damme W J van, Grind W A van de, 1996 "Non-visual information in structure-from-motion" Vision Research $363119-3127$

Dijkstra T M, Cornilleau-Pérès V, Gielen C C, Droulez J, 1995 "Perception of three-dimensional shape from ego- and object-motion: comparison between small- and large-field stimuli" Vision Research $35453-462$

Fischer B, Boch R, 1981 "Enhanced activation of neurons in prelunate cortex before visually guided saccades of trained rhesus monkeys" Experimental Brain Research 44129 - 137

Gibson J J, 1966 The Senses Considered as Perceptual System (Boston, MA: Houghton Mifflin)

Harrington T L, 1965 "Adaptation of humans to colored split-field glasses" Psychonomical Science $371-72$

Harris C S, 1965 "Perceptual adaptation to inverted, reversed and displaced vision" Psychological Review $92419-444$

Held R, Freedman S J, 1963 "Plasticity in human sensorimotor control" Science $142455-462$

Hepler N, 1968 "Colour: a motion contingent after-effect" Science 162 376-377

Hurley S L, 1998 Consciousness in Action (London: Harvard University Press)

Jameson D, Hurvich L M, 1978 “Dichromatic color language: 'Reds' and 'Greens' don't look alike but their colors do" Sensory Processes 2 146-155

Kohler I, 1962 "Experiment with goggles" Scientific American 206(5) 62-72

Leppmann P K, Wieland A B, 1966 "Visual distortion with two-colored spectacles" Perceptual and Motor Skills $231043-1048$

McCollough C, 1965a "Color adaptation of edges-detectors in the human visual system" Science $1491115-1116$

McCollough C, 1965b "The conditioning of color perception" American Journal of Psychology 78 $362-378$

Mayhew J, 1973 "After-effects of movement contingent on direction of gaze" Vision Research 13 $877-880$

Milner A D, Goodale M, 1995 The Visual Brain in Action (Oxford: Oxford University Press)

Myin E, 2001 "Color and the duplication assumption" Synthese 129(1) $61-77$

O'Regan J K, Noë A, 2001 "A sensorimotor account of vision and visual consciousness" Behavior and Brain Science $24939-1011$

Pelli D G, 1997 "The Video Toolbox software for visual psychophysics: transforming numbers into movies" Spatial Vision $10437-442$

Rayner K, 1984 "Visual selection in reading, picture perception and visual search. A tutorial review" Attention and Performance $1067-96$

Reppas J B, Ursey W M, Reid R C, 2002 "Saccadic eye movements modulate visual responses in the LGN" Neuron $35961-974$

Rock I, 1966 The Nature of Perceptual Adaptation (New York: Basic Books)

Rogers S, Rogers B J, 1992 "Visual and nonvisual information disambiguate surfaces specified by motion parallax" Perception \& Psychophysics 52 446-452 
Roorda A, Williams D R, 1999 "The arrangement of the three cone classes in the living human eye" Nature $397520-522$

Skaff S, Arbel T, Clark J J, 2002 "Active bayesian color constancy with non-uniform sensors" 16th International Conference on Pattern Recognition volume 2, pp 681-684

Tolias A S, Moore T, Smirnakis S M, Tehovnik E J, Siapas A G, Schiller P H, 2001 "Eye movements modulate visual receptive fields of V4 neurons" Neuron $29757-767$

Ungerleider L G, Mishkin M, 1982 "Two cortical visual systems", in Analysis of Visual Behavior Eds D J Ingle, M A Goodale, R J W Mansfield (Cambridge, MA: MIT Press) pp 549-586

Welch R B, 1974 "Research on adaptation to rearranged vision: 1966-1974" Perception 3367 - 392

Wexler M, Panerai F, Lamouret I, Droulez J, 2001 "Self-motion and the perception of stationary objects" Nature $40985-88$ 


\section{PERCEPTION}

VOLUME 352006

www.perceptionweb.com

Conditions of use. This article may be downloaded from the Perception website for personal research by members of subscribing organisations. Authors are entitled to distribute their own article (in printed form or by e-mail) to up to 50 people. This PDF may not be placed on any website (or other online distribution system) without permission of the publisher. 